\title{
Interdisciplinaridade e IHC: Possibilidades no Contexto da Licenciatura em Computação
}

\author{
Igor Felipe Gomes \\ de Lima
}

\author{
Matheus Carvalho \\ de Melo
}

\author{
Wellington Eugenio \\ da Silva
}

\author{
Taciana Pontual \\ Falcão
}

\author{
Departamento de Computação, \\ Universidade Federal Rural de Pernambuco, Recife/PE-Brasil \\ Igor7@outlook.com, Teteretu@yahoo.com,WellingtonEugenio@gmail.com, Taciana.Pontual@ufrpe.br
}

\section{RESUMO}

A interdisciplinaridade é vista cada vez mais como um componente fundamental para a educação em todos os níveis. Neste artigo, analisamos opiniões, motivações e barreiras, percebidas por professores, para a disseminação de práticas interdisciplinares; e apresentamos práticas existentes em nossos cursos de graduação na área de Computação. Em particular, focamos no potencial da Interação Humano-Computador como uma área de conhecimento transversal e em como isso pode ser melhor explorado em cursos de Licenciatura em Computação. Consideramos os desafios inerentes às diferentes possibilidades para promover a interdisciplinaridade e a necessidade de repensar a estrutura atual da educação superior.

\section{PALAVRAS-CHAVE}

Interdisciplinaridade, Licenciatura em Computação, Interação Humano-Computador, Desenvolvimento de Software.

\section{Introdução}

Partindo-se da perspectiva de que o profissional de Computação deve ser capaz de resolver problemas de quaisquer áreas do conhecimento de forma eficaz e eficiente, é necessário também que este saiba interagir com profissionais de outras áreas a fim de produzir soluções realmente adequadas e completas. Um exemplo da indústria são as equipes de desenvolvimento de software, que normalmente reúnem profissionais de diversas formações.

Permission to reproduce or distribute, in whole or in part, material extracted from this work, verbatim, adapted or remixed, as well as the creation or production from the content of such work, is granted without fee for non-commercial use, provided that the original work is properly credited.

IHC 2019 - Workshop sobre Educação em IHC (WEIHC), Outubro 21-25, 2019, Vitória, Brasil. In Anais Estendidos do XVIII Simpósio Brasileiro sobre Fatores Humanos em Sistemas Computacionais. Porto Alegre: SBC. (C) 2019 by the author(s), in accordance with the terms of the Creative Commons Attribution-NonCommercial 4.0 International Public License (CC BY-NC 4.0).
Logo, se a interdisciplinaridade se faz necessária para a prática profissional, então deve ser vivenciada durante a formação.

De forma mais ampla, a interdisciplinaridade é citada na Base Nacional Comum Curricular ${ }^{1}$ entre as ações necessárias à aprendizagem integradora e transversal. Entretanto, implementar a interdisciplinaridade não é um processo simples, dada a própria formação docente e a organização curricular fragmentadas. Assim, para que a interdisciplinaridade seja concretizada nas instituições educacionais, é necessário um esforço conjunto de todos os atores envolvidos no processo de ensino-aprendizagem [1].

Em cursos de Computação, a disciplina de Interação HumanoComputador (IHC) possui uma relação com várias outras disciplinas ligadas ao desenvolvimento de software, tornando-se muito propícia à interdisciplinaridade, como é possível constatar analisando-se os anais passados do WEIHC [9, 10, 12]. Percebe-se, porém, que as iniciativas apresentadas ainda são pontuais, e com pouco impacto a nível administrativo ou curricular.

Somando-se ao esforço em promover a interdisciplinaridade no ensino superior, com foco na disciplina de IHC no contexto de desenvolvimento de software, este artigo apresenta: um breve diagnóstico da visão dos docentes do Departamento de Computação da Universidade Federal Rural de Pernambuco (UFRPE) acerca da interdisciplinaridade; algumas possibilidades já em andamento em cursos de Computação na região; uma proposta de ementa de referência de IHC para cursos de licenciatura em computação (LC); uma proposta interdisciplinar específica para as disciplinas de IHC e Projeto de Desenvolvimento de Software Educacional (PDSE), em um curso de LC. O artigo é resultado de um trabalho realizado na disciplina de Prática de Ensino de IHC, em que são discutidos aspectos relacionados à educação em IHC.

\section{Interdisciplinaridade: Diagnóstico \& Desafios}

A interdisciplinaridade provê formação e visão mais globais ao estudante, que adquire a habilidade de articular e contextualizar os conhecimentos ao distanciar-se da fragmentação conteudista em que tradicionalmente se pautam os currículos em escolas e

\footnotetext{
${ }^{1}$ http://basenacionalcomum.mec.gov.br/
} 


\section{WEIHC'19, Outubro, 2019, Vitória, ES - Brasil}

universidades. [8] apud [1] cita três esferas de interdisciplinaridade: curricular, didática e pedagógica. A interdisciplinaridade curricular localiza-se no âmbito administrativo, definindo currículos, objetivos e programas das disciplinas. A interdisciplinaridade didática compreende o planejamento para integrar os conteúdos programáticos das disciplinas. A interdisciplinaridade pedagógica trata de como a prática pedagógica interdisciplinar ocorre na sala de aula. Apesar dos benefícios das abordagens interdisciplinares, ainda há muitas barreiras no cotidiano das instituições educacionais nessas três esferas [1, 2]. A própria formação específica dos docentes, a distância de linguagem e perspectivas entre disciplinas, e a falta de tempo e espaços para inovar nas instituições educacionais são alguns exemplos dessas dificuldades.

Como constataram [7], a formação acadêmica de acordo com uma concepção fragmentada pode gerar no professor insegurança em pensar um trabalho coletivo e interdisciplinar. Em pesquisas com professores da educação básica, [2] identificaram três categorias de dificuldades enfrentadas por eles para desenvolver atividades interdisciplinares: fragmentação disciplinar; diálogo com colegas e gestores; problemas de interesse e conhecimento. Também em pesquisas com professores, [1] classificaram em três níveis os impeditivos para a interdisciplinaridade declarados pelos participantes: entendimento da construção do conhecimento científico; organização do trabalho coletivo; e prática pedagógica. Percebe-se que os achados das duas pesquisas são bastante semelhantes, embora apresentados com palavras distintas.

\subsection{Diagnóstico sobre Interdisciplinaridade no Departamento de Computação}

Dados os resultados relativos à educação básica, buscamos diagnosticar a prática da interdisciplinaridade no ensino superior, mais precisamente no Departamento de Computação da UFRPE, a fim de pensar em propostas para disseminar a interdisciplinaridade da IHC com outras disciplinas dos cursos.

Os questionários de $[1,2]$ serviram de base a para a composição de um novo questionário online ${ }^{2}$ com escalas Likert de 1 a 5 versando sobre: opiniões acerca da interdisciplinaridade; práticas realizadas; formação e competência para praticar a interdisciplinaridade, e dificuldades relacionadas a tempo, trabalho cooperativo e recursos, entre outras.

O questionário foi distribuído online e respondido (de maneira anônima) por 27 dos 47 docentes do departamento (todos com formação em Computação). $88 \%$ classificaram a importância da interdisciplinaridade no ensino superior nos níveis 4 e 5 (em uma escala de muito baixo a muito alto), porém a prática da interdisciplinaridade se dá em níveis menores, com $66 \%$ das respostas nos níveis 3 e 4 (numa escala de não pratico ou pratico muito pouco a pratico muito). Considerando o conteúdo das disciplinas que lecionam, $44 \%$ consideram que a maior parte do conteúdo pode ser trabalhado interdisciplinarmente. Sobre o tempo

\footnotetext{
${ }^{2}$ Disponível em: https://forms.gle/sq6gNqmviSkvuwoF6

https://projetao.ranoya.com/
}

\section{Lima et al.}

disponível para interdisciplinaridade, 77,7\% classificaram entre os níveis 3 e 5 , enquanto $22,2 \%$ classificaram entre os níveis 1 e 2 (pouco ou muito pouco tempo). Dados relacionados à formação acadêmica também foram levantados: $81,5 \%$ classificaram como pouca ou muito pouca a vivência da interdisciplinaridade durante a própria formação acadêmica e $88,9 \%$ relataram que a universidade os capacita pouco ou muito pouco a respeito. Apesar disso, 55,6\% se consideram medianamente preparados para lidar com a interdisciplinaridade, enquanto $22,2 \%$ se consideram muito preparados e $14,8 \%$ pouco preparados. Em relação ao tempo de planejamento para a interdisciplinaridade, $74 \%$ classificaram entre os níveis 3 e 5 , enquanto $26 \%$ classificaram no nível 2 (pouco ou muito pouco tempo). Além disso, 81,4\% acham que faltam recursos adequados. Por fim, a maior parte dos docentes está muito disposta a cooperar com os colegas para promover a interdisciplinaridade, porém existe uma incompatibilidade razoável de horários que impede tal cooperação.

Percebem-se algumas semelhanças com os achados de [1, 2], principalmente em termos da dificuldade de se organizar um trabalho coletivo que supere a fragmentação disciplinar e da falta de formação para ensinar de maneira interdisciplinar (prática pedagógica). Por outro lado, existe uma boa disposição e motivação em se aumentar as práticas interdisciplinares, além de uma opinião de que essas práticas são relevantes e importantes.

Sugestões dadas pelo docentes em campo aberto incluem: disciplinas oferecidas em conjunto; maior interação com colegas de outros departamentos que atuam nos cursos de Computação; formação pedagógica voltada para a interdisciplinaridade; interdisciplinaridade prevista e considerada no Projeto Pedagógico do Curso e nas ementas das disciplinas; flexibilidade de horários; conscientização dos docentes; reuniões de planejamento; mudança estrutural do sistema educacional.

\section{Experiências Interdisciplinares Inspiradoras}

Duas experiências interdisciplinares de destaque nas universidades da região têm sido referência para outras iniciativas, e confirmam o interesse pela interdisciplinaridade.

Tendo a interdisciplinaridade como um dos seus valores, o Centro de Informática da UFPE formatou a disciplina de Projeto de Desenvolvimento, apelidada de Projetão ${ }^{3}$, para ser ofertada de forma integrada a alunos de Ciência e Engenharia da Computação, Psicologia e Design, entre outros cursos interessados. A disciplina tem como objetivo o desenvolvimento de um sistema de computação que use conceitos aprendidos nos semestres anteriores do curso, representando um marco do fím do ciclo básico, com ênfase em empreendedorismo e inovação. Um dos principais objetivos do Projetão é a integração e mobilidade entre os cursos. As equipes têm, obrigatoriamente, estudantes de diferentes cursos e são mentoreadas por seis consultores (entre professores e monitores). A disciplina segue metodologias ágeis de 


\section{Interdisciplinaridade e IHC: Possibilidades no Contexto da Licenciatura em Computação}

desenvolvimento de software orientadas à inovação e baseadas em desafios. As equipes funcionam como startups e apresentam seus produtos em forma de pitch em um "demo day". A avaliação dos estudantes leva em consideração vários aspectos do desenvolvimento dos projetos e trabalho em equipe. Este formato de disciplina tem motivado os estudantes a buscarem solucionar problemas reais de maneira interdisciplinar e até mesmo levar adiante as startups nascidas na disciplina.

Na UFRPE, o Projeto TEIA - Técnicas de Educação Inovadora com Metodologias Ativas - propõe a integração de duas ou mais disciplinas em um mesmo horário e local. As equipes são necessariamente formadas por alunos matriculados nas diversas disciplinas, e os professores responsáveis atuam juntos como mediadores, porém cada um com sua expertise particular. As aulas expositivas são reduzidas a um mínimo para que os estudantes se dediquem a planejar seus ciclos de desenvolvimento de projeto (sprints) e as entregas correspondentes pelas quais são avaliados. A avaliação de cada estudante é de acordo com os conteúdos da disciplina em que está matriculado. A aprendizagem é baseada em problemas; e os estudantes podem escolher desafios de um amplo conjunto pré-definido pelos professores, que buscam orientar seus alunos a construírem o conhecimento esperado através do desenvolvimento de habilidades de autonomia no aprendizado e trabalho em equipe. O Projeto TEIA já foi executado com as disciplinas de IHC e Desenvolvimento de aplicações web; e Empreendedorismo e Inovação.

As experiências descritas confirmam o interesse dos docentes em desenvolver a interdisciplinaridade no ensino superior. No entanto, ambas ainda dependem muito das iniciativas e da persistência dos docentes para superar barreiras burocráticas do sistema educacional baseado em créditos e fragmentado em disciplinas. Um caminho complementar que pode fortalecer tais iniciativas seria trabalhar no nível institucional, como sugerido por alguns docentes na pesquisa realizada no nosso departamento, ao prever a interdisciplinaridade nas ementas, conteúdos programáticos e projetos pedagógicos dos cursos.

\section{Proposta}

A proposta aqui apresentada procura trabalhar a interdisciplinaridade no nível do conteúdo programático das disciplinas. Considerando o escopo da disciplina de Prática de Ensino de IHC, na qual foi realizado este trabalho, o foco da proposta é na relação da disciplina de IHC com outras disciplinas do curso de Licenciatura em Computação da UFRPE. Para fazer tal análise de convergência entre os conteúdos de IHC e outras disciplinas fez-se necessário, primeiramente, ter uma ementa de referência para IHC em cursos de LC.
WEIHC'19, Outubro, 2019, Vitória, ES - Brasil

\subsection{Proposta de Ementa de Referência de IHC para Cursos de LC}

A motivação para esta etapa do trabalho surgiu a partir da análise do artigo "Currículos de IHC no Brasil: Panorama Atual e Perspectivas" [4]. O artigo apresenta ementas de IHC para os cursos de Ciência da Computação, Sistemas de Informação e Engenharia da Computação; resultantes das decisões de grupos de trabalho formados durante o WEIHC 2014. Nota-se, portanto, que ficou uma lacuna a ser preenchida em relação ao curso de Licenciatura em Computação. Descrevemos abaixo as etapas realizadas para preencher essa lacuna.

Inicialmente foi feita uma busca no Cadastro Nacional de Cursos e Instituições de Educação Superior ${ }^{4}$, disponível no site do Ministério da Educação, para identificar instituições que ofertam o curso de Licenciatura em Computação. Depois foi feita a busca pelos projetos pedagógicos dos cursos nos sites das respectivas instituições de ensino, de onde foram extraídas as ementas de IHC. Nossa amostra conteve 26 ementas de IHC, sendo 11 de universidades federais, 9 de institutos federais e 6 de universidades estaduais: UFRPE, UFPR, UFERSA, UFBA, UFRA, UFJF, UFRR, UFTO, UFPI, UFPA, UNIVASF, IFPB, IFTM, IFRN, IFBA, IF Sertão-PE, IFPE, IF Farroupilha, IFMS, IFPR, UPE, CESUPA, UESPI, UECE, UENP, UDESC.

As ementas foram lidas em grupo nos encontros da disciplina de Prática de Ensino de IHC, e os tópicos citados e a frequência com que se repetiam foram registrados em post-its. Em seguida, esses tópicos foram agrupados em categorias por similaridade semântica. A seleção dos tópicos foi feita com base na frequência e relevância dos mesmos, evitando repetições e ambiguidades. Dois livros de referência na área de IHC no Brasil foram consultados para fins validação e complementação da ementa: Interação HumanoComputador [3]; e Ergonomia e Usabilidade [6]. A ementa de referência de IHC para cursos de LC no Brasil é apresentada a seguir:

Usabilidade e experiência do usuário. Ergonomia. Acessibilidade. Estilos e dispositivos de interação: interfaces gráficas e manipulação; interfaces web; dispositivos móveis; interfaces baseadas em toque e gestos; interfaces por comando de voz. Padrões para interfaces. Diretrizes de design de interfaces. Projeto de Interfaces e Interação. Processo de design centrado no usuário. Modelagem de tarefas. Modelagem de Interfaces. Ferramentas para construção de interface. Prototipação de interfaces. Fatores humanos: aspectos culturais, sociais e éticos; aspectos cognitivos e perceptivos; psicologia cognitiva aplicada. Avaliação de interfaces: por inspeção e por observação. Engenharia semiótica. Comunicabilidade.

Percebe-se que a ementa construída a partir de cursos de LC tem tópicos muito semelhantes à ementa sugerida por [4] para cursos de Ciência da Computação e que, portanto, não há questões

\footnotetext{
${ }^{4}$ http://emec.mec.gov.br
} 


\section{WEIHC'19, Outubro, 2019, Vitória, ES - Brasil}

particulares relacionadas à licenciatura em termos de ementa. No entanto, o professor deve, de preferência, relacionar os tópicos da ementa com contextos e tecnologias educacionais.

\subsection{IHC \& Projeto de Desenvolvimento de Software Educacional}

Tendo construído uma ementa de referência para IHC em cursos de LC, foi realizada uma análise das ementas das demais disciplinas do curso de LC da UFRPE, disponíveis do Projeto Pedagógico do Curso $^{5}$. Nessa análise, surgiu a motivação de aproximar a IHC da disciplina de Projeto de Desenvolvimento de Software Educacional (PDSE), a ser ofertada pela primeira vez no segundo semestre de 2019, como parte da nova matriz do curso ${ }^{6}$. PDSE é uma disciplina derivada da disciplina de Projeto de Desenvolvimento de Software, fortemente baseada em conceitos da Engenharia de Software, porém com um viés prático e aplicado, objetivando a implementação de um produto de software. O diferencial de PDSE é a aplicação do software em contexto educacional. Com isso, torna-se uma disciplina complexa que precisaria levar em conta as necessidades do usuário, heurísticas de interface e heurísticas voltadas a softwares educacionais.

Entretanto, percebeu-se que a ementa (transcrita abaixo) de PDSE no curso de LC da UFRPE não contém nenhum tópico de IHC ou de Educação, e apenas menciona superficialmente que a "multidisciplinaridade" deve ser contemplada:

Instanciação de um processo de desenvolvimento de software: fluxo, atividades, papéis e produtos de trabalho. Desenvolvimento de projeto multidisciplinar em equipe. Implementação de um produto de software. Uso de padrões de projeto e de codificação de software. Realização de testes automatizados de software.

Assim, a clara prevalência da Engenharia de Software "pura" no decorrer da disciplina de PDSE cria um forte viés que provavelmente influenciará o desenvolvimento do software educacional, possivelmente negligenciando aspectos importantes de IHC e pedagógicos. Percebe-se que os objetivos da disciplina, também constantes no Projeto Pedagógico, tampouco contemplam aspectos de IHC:

- Prover aos estudantes a oportunidade de desenvolver um programa de software com fins explicitamente educacionais;

- Orientar os estudantes no desenvolvimento de um software de acordo com um processo formal;

- Orientar os estudantes na integração de questões didáticas e pedagógicas ao processo de desenvolvimento de software.

Acreditamos que uma abordagem equilibrada interdisciplinar poderia gerar um produto mais adequado, ao mesmo tempo em que proveria uma vivência mais completa para os estudantes. As

\section{Lima et al.}

atividades tradicionalmente realizadas em disciplinas de projeto de desenvolvimento de software podem ser complementadas e integradas com diversos conceitos e técnicas fundamentais da IHC. A seguir, cada fase da disciplina de PDSE, começando pela concepção da ideia do software até a última fase de testes utilizando a versão final, é apresentada e relacionada com as técnicas de IHC. As técnicas foram escolhidas a partir dos tópicos da ementa proposta de IHC, mas também considerando-se as restrições de uma disciplina de 60 hora/aula e cujo foco principal não é a IHC. Trata-se, portanto, de um conteúdo extenso, aliado à necessidade de desenvolvimento de outras competências ligadas ao desenvolvimento de software em equipes. Na matriz curricular do curso, a disciplina de IHC é ministrada antes de PDSE. Assim, podemos assumir que os estudantes já possuem o conhecimento mais amplo de IHC a ser aplicado ao longo do desenvolvimento do software.

4.2.1 Concepção da Ideia do Projeto. No contexto da disciplina de PDSE, sugerimos o uso de personas para caracterizar o perfil do usuário, traçando cenários para projetar o software de maneira focada no público-alvo. Um software educacional tem características muito peculiares a depender do seu público-alvo e, no contexto restrito de uma disciplina, pode ser difícil envolver o usuário no processo, principalmente se forem crianças. O uso de personas e cenários facilita o design centrado no usuário ao diminuir a necessidade de contato com o mesmo.

4.2.2 Especificação de Requisitos Funcionais e Não Funcionais. Nesta fase, podem ser usadas técnicas de modelagem de tarefas do usuário (sugerimos Análise da tarefa). Além disso, conceitos de IHC devem ser inseridos nas questões não funcionais do produto: Considerar os aspectos sociais, éticos, cognitivos e perceptivos dos usuários, ainda tendo como referência as personas e cenários, de acordo com suas necessidades, sempre focando no que é preciso para a melhor adaptação do usuário à interface proposta; Faz-se necessária a verificação dos aspectos educacionais do software, incluindo a abordagem pedagógica será adotada para desenvolver o conteúdo (por exemplo, um enfoque behaviorista ou construtivista); Considerar nível de dificuldade do conteúdo educacional do software, a fim de ter uma evolução cognitiva progressiva do assunto abordado; Procurar conceituar e aplicar progressivamente o conteúdo educacional do software, de uma forma que o usuário possa reconhecer as ações que tenha que fazer e não as memorizar; Ter em mente quais são os objetivos em relação à aprendizagem.

4.2.3 Projeto e Modelagem. Essa fase trata da estrutura que vai servir de guia para todo o projeto. Em termos de IHC, deve-se definir um padrão de projeto relacionado a cores, estilo de fonte, ícones, e elementos de interação e visualização. Sugerimos o uso de moodboard (para padrões de cores, iluminação, estilo visual de como deve ser desenvolvido no software) e mockups (protótipo para estruturar o desenho e o fluxo das telas). É importante fazer uso de elementos multimídia que despertem o interesse pelo

\footnotetext{
${ }^{5}$ www.lc.ufrpe.br//br/content/projeto-pedagógico-do-curso

${ }^{6} \mathrm{http} / /$ www.lc.ufrpe.br/br/content/matriz-curricular-partir-de-20182
} 


\section{Interdisciplinaridade e IHC: Possibilidades no Contexto da Licenciatura em Computação}

conteúdo educacional. Além disso, aplicar heurísticas de interface no momento da concepção de telas é importante para que no futuro o software possa ser validado cumprindo essas heurísticas. Em se tratando de software educacional, devem ser consideradas, além das heurísticas de interface (como as heurísticas de Nielsen [3]), as heurísticas com foco educacional, como $[5,11]$.

4.2.4 Desenvolvimento e Implementação do Software. Durante a implementação, verificar se os padrões de IHC estão sendo aplicados. Por exemplo, para garantir a acessibilidade do software, que tem uma importância ainda maior em se tratando de um software educacional. Além disso, garantir um tratamento de erros com mensagens adequadas do ponto de vista do usuário, informando o erro em uma linguagem adequada, com a causa, a consequência e como o usuário pode agir dado o problema apresentado.

4.2.5 Testes do Sistema. Os testes automatizados de software devem ser combinados a avaliações de usabilidade para garantir que o produto atenda as necessidades do usuário. Sugerimos uma avaliação heurística para verificar se a fase de desenvolvimento e implementação conseguiu concretizar as heurísticas definidas na fase de projeto e modelagem. De forma complementar, pode-se também realizar testes de usabilidade com representantes do público-alvo. Se preciso, propor um plano de melhorias com base nos testes realizados.

A Tabela 1 apresenta o mapeamento entre as fases do desenvolvimento do software como previsto na disciplina de PDSE, os tópicos relacionados da ementa consolidada de IHC na LC, e os aspectos educacionais que devem ser considerados.

É importante destacar que a integração proposta pode acontecer independentemente da metodologia de desenvolvimento de software adotada. Ou seja, se o professor optar por metodologias ágeis, essas fases podem ser executadas iterativamente.

\subsection{Avaliação da Proposta}

Visto que a disciplina de PDSE ainda não foi ofertada, a proposta de interdisciplinaridade de IHC com PDSE foi discutida com o professor atualmente responsável pela disciplina de Projeto de Desenvolvimento de Software, do curso de Bacharelado em Ciência da Computação.

Em entrevista, o professor aprovou a proposta, que achou não somente viável, mas essencial. Ele relatou sua motivação em usar a interdisciplinaridade para melhorar a compreensão e aumento de qualidade de desenvolvimento de software com o uso de IHC, principalmente na fase de prototipação de software. Além de IHC, o professor acredita que conteúdos de inovação e empreendedorismo devem ser integrados à disciplina de projeto de desenvolvimento de software. De fato, esses aspectos podem ser integrados de diferentes maneiras: incentivando os estudantes a registrarem os produtos; promovendo apresentações do produto a empresas da região; encorajando a criação de startups na área de tecnologias educacionais, entre outros.
WEIHC’19, Outubro, 2019, Vitória, ES - Brasil

O professor também acredita que o formato da disciplina deve cada vez mais se afastar do tradicional e aproximar-se de experiências como o "Projetão" e o Projeto TEIA, relatados na seção 3, com alunos e professores de várias áreas de conhecimento trabalhando juntos em um mesmo projeto. Entretanto, ele reconhece que esse formato traz dificuldades práticas pois demanda alinhamento de horários de disciplinas, cursos, professores, necessidade de espaços maiores e planejamento conjunto.

\begin{tabular}{|c|c|c|}
\hline Fases PDSE & Técnica de IHC & $\begin{array}{l}\text { Aspectos } \\
\text { Educacionais }\end{array}$ \\
\hline $\begin{array}{l}\text { Concepção da } \\
\text { Ideia }\end{array}$ & Personas; Cenários & $\begin{array}{l}\text { Conteúdo } \\
\text { educacional; } \\
\text { Características do } \\
\text { público-alvo }\end{array}$ \\
\hline $\begin{array}{l}\text { Especificação de } \\
\text { Requisitos }\end{array}$ & $\begin{array}{l}\text { Personas; Cenários } \\
\text { Análise da tarefa }\end{array}$ & $\begin{array}{l}\text { Objetivos de } \\
\text { aprendizagem; } \\
\text { Teoria de } \\
\text { aprendizagem }\end{array}$ \\
\hline $\begin{array}{l}\text { Projeto e } \\
\text { Modelagem }\end{array}$ & $\begin{array}{l}\text { Moodboard; } \\
\text { Mockups; } \\
\text { Heurísticas de } \\
\text { interface }\end{array}$ & $\begin{array}{l}\text { Heurísticas para } \\
\text { software } \\
\text { educacional }\end{array}$ \\
\hline $\begin{array}{l}\text { Implementação do } \\
\text { Software }\end{array}$ & $\begin{array}{l}\text { Acessibilidade; } \\
\text { Heurísticas de } \\
\text { interface }\end{array}$ & - \\
\hline Testes do Sistema & $\begin{array}{l}\text { Avaliação } \\
\text { heurística; Teste } \\
\text { de usabilidade }\end{array}$ & $\begin{array}{l}\text { Avaliação com } \\
\text { público-alvo }\end{array}$ \\
\hline
\end{tabular}

Tabela 1: Relação entre fases de desenvolvimento do software, tópicos de IHC e aspectos educacionais.

\section{Considerações Finais}

Cada vez mais, docentes e discentes percebem a importância da abordagem interdisciplinar no ensino superior. As convergências entre disciplinas são claras em muitos casos, porém a vontade de implantar projetos interdisciplinares esbarra na burocracia e nas amarras do sistema baseado em créditos e fragmentação curricular. Por outro lado, os docentes têm pouca formação e incentivo institucional para implementar a interdisciplinaridade, além das dificuldades mais pragmáticas relacionadas a falta de tempo, recursos e necessidade de organização conjunta com colegas.

Apesar de todos esses fatores, é possível mapear várias iniciativas de docentes que tentam superar as barreiras e realizar atividades interdisciplinares. A IHC em particular é uma disciplina que dialoga com muitas outras em cursos de Computação e, portanto, é propícia a interações frutíferas. Neste artigo, apresentamos um 


\section{WEIHC'19, Outubro, 2019, Vitória, ES - Brasil}

trabalho de pesquisa fruto da disciplina de Prática de Ensino de IHC, que focou nas possibilidades de interdisciplinaridade da IHC em cursos de Licenciatura em Computação, que têm agregado o desafio extra de integrar aspectos educacionais às soluções de software.

Apresentamos alternativas de interdisciplinaridade que unem diferentes cursos em uma única disciplina de desenvolvimento de software, e que unem diferentes disciplinas em um mesmo curso. Porém, ao mesmo tempo em que o sucesso dessas iniciativas confirma a importância da interdisciplinaridade, ainda são iniciativas que de alguma forma desviam-se do sistema educacional como é formalmente colocado, encontrando caminhos alternativos de implementação. É importante pensar a interdisciplinaridade também a partir dos projetos pedagógicos dos cursos, ementas e conteúdos programáticos das disciplinas. Assim, apresentamos neste artigo uma proposta para a disciplina de Projeto de Desenvolvimento de Software Educacional (PDSE), que deveria contemplar em seu programa não só aspectos de IHC como também pedagógicos. Um trabalho semelhante ao feito com IHC e PDSE pode ser realizado em todo o projeto pedagógico do curso, atualizando os conteúdos programáticos das disciplinas para indicar pontos de interdisciplinaridade oficialmente.

A interdisciplinaridade nas ementas, por outro lado, gera um desafio para os docentes visto que a sua própria formação é baseada no currículo fragmentado. Ou seja, um professor de Engenharia de Software que ministre PDSE pode não ter nenhum conhecimento de IHC e sentir-se inseguro na disciplina. Isso nos leva novamente à questão mais ampla da estrutura dos cursos de graduação, com disciplinas muito bem separadas e professores com expertises correspondentes. No entanto, em cursos de Computação, tem sido cada vez mais gritante o problema de tal abordagem, visto que o desenvolvimento de tecnologias sempre envolve mais de uma disciplina. A IHC, em particular, é um conhecimento transversal em muitos casos. Nos dias atuais, fica a cargo do aluno conseguir unir os conhecimentos fragmentados para construir competência e visão mais globais na sua trajetória profissional.

O estudo aqui apresentado, portanto, confirma a necessidade de se repensar a estrutura dos cursos de graduação no Brasil. Começar por "institucionalizar a interdisciplinaridade", como apresentado neste trabalho, pode ser um gatilho neste sentido. Para a comunidade de IHC, fica o desafio de se repensar como a IHC deveria figurar em cursos de Computação, indo muito além do formato de uma única disciplina básica nos cursos.

\section{AGRADECIMENTOS}

Agradecemos a todos os professores participantes das diferentes fases da pesquisa.

\section{REFERÊNCIAS}

[1] T.G.S. Augusto e A.M.A. Caldeira. (2016). Dificuldades para a implantação de práticas interdisciplinares em escolas estaduais, apontadas por professores da área de ciências da natureza. Investigações em Ensino de Ciências, 12(1), 139154.

\section{Lima et al.}

[2] L.A.B. Avila, D.V. Matos, A.L.P. Thiele, e M.G. Ramos (2017). A interdisciplinaridade na escola: dificuldades e desafios no ensino de ciências e matemática. Signos, Lajeado, 38(1), 9-23.

[3] S.D.J. Barbosa e B.S. Silva. 2010. Interação humano-computador (1nd. ed.). Elsevier Editora Ltda, Rio de Janeiro.

[4] C. Boscarioli et al. (2014). Currículos de IHC no Brasil: panorama atual e perspectivas. Em: Anais do XXII Workshop sobre Educação em Computação.

[5] O. O. Brito Junior e Y. P. C. Aguiar (2018). Taxonomia de Critérios para Avaliação de Software Educativo - TaCASE. Em: Anais do Simpósio Brasileiro de Informática na Educação.

[6] W. Cybis; A.H. Betiol; R. Faust. (2017). Ergonomia e usabilidade: conhecimentos, métodos e aplicações. Novatec editora.

[7] A.B. Kleiman e S.E. Moraes. (1999). Leitura e interdisciplinaridade: tecendo redes nos projetos da escola. Campinas: Mercado das Letras.

[8] Y. Lenoir. (2001). Didática e interdisciplinaridade: uma complementaridade necessária e incontornável. Em: I.C.A Fazenda (org). Didática e interdisciplinaridade. Campinas: Papirus.

[9] A.B.S. Marques e Á.H.S. Melo. (2015). Relato de experiência no ensino de IHC em nível de graduação através de uma abordagem interdisciplinar. VI Workshop sobre Ensino de IHC - XIV Simpósio Brasileiro sobre Fatores Humanos em Sistemas Computacionais.

[10] E.S. Matos. (2013). Integração curricular por meio da prática de ensino interdisciplinar em IHC. IV Workshop sobre Ensino de IHC. 25-30.

[11] P. Valle, R. Vilela, P. Parreira, A. Inocêncio. (2013) HEDEG - Heurísticas para Avaliação de Jogos Educacionais Digitais. Em: Anais do Nuevas Ideas en Informática Educativa - TISE.

[12] L.A.M. Zaina e A. Álvaro (2013). O ensino de IHC potencializando ideias empreendedoras. Em: Anais do Workshop de Educação em IHC - WEIHC 2013. 